ज्) FRANÇAISE

$>\mathrm{DE}$

띠 PÉDAGOGIE

\section{Revue française de pédagogie}

Recherches en éducation

168 | juillet-septembre 2009

Enseignement et apprentissages, entre psychologie et didactiques

\title{
L'activité didactique empêchée : outil d'intelligibilité de la pratique enseignante en milieu difficile
}

Cancelled teaching activities: a tool to understand teaching practices in difficult areas

La actividad didáctica obstaculizada : herramienta de inteligibilidad de la práctica docente en un entorno difícil

Verhinderte pädagogische Aktivität: ein Werkzeug zur Verständigung der Lehrpraktiken in Problemgebieten

Nathalie Monnier et Chantal Amade-Escot

\section{OpenEdition}

Journals

Édition électronique

URL : http://journals.openedition.org/rfp/1750

DOI : 10.4000/rfp. 1750

ISSN : 2105-2913

Éditeur

ENS Éditions

Édition imprimée

Date de publication : 1 juillet 2009

Pagination : $59-73$

ISBN : 978-2-7342-1172-3

ISSN : 0556-7807

\section{Référence électronique}

Nathalie Monnier et Chantal Amade-Escot, «L'activité didactique empêchée : outil d'intelligibilité de la pratique enseignante en milieu difficile », Revue française de pédagogie [En ligne], 168 । juilletseptembre 2009, mis en ligne le 01 juillet 2013, consulté le 01 mai 2019. URL : http:// journals.openedition.org/rfp/1750; DOI : 10.4000/rfp.1750 


\section{L'activité didactique empêchée : outil d'intelligibilité de la pratique enseignante en milieu difficile}

Nathalie Monnier et Chantal Amade-Escot

Considérant la transmission des savoirs au cœur de l'activité enseignante, cette étude discute la compatibilité de deux orientations de recherche: la didactique et la clinique de l'activité. À partir d'un prototype méthodologique visant à explorer "l'activité didactique empêchée » de professeurs d'éducation physique et sportive (EPS) enseignant en milieu difficile, elle met en évidence les conditions à la fois théoriques et méthodologiques qui permettent d'importer au sein d'un dispositif d'observation didactique un dispositif d'instruction au sosie. II s'agit d'accéder à la part empêchée de l'activité didactique, en identifiant les tensions et les conflits de la pratique enseignante en milieu difficile susceptibles d'expliquer la minoration des savoirs maintes fois constatée par la littérature. Cet article s'appuie sur des extraits relatifs à une séance d'enseignement du badminton en EPS en lycée professionnel. Il met en évidence que l'activité didactique est contrainte par la poursuite d'enjeux multiples qui s'imposent à l'enseignant afin que puisse aboutir l'enjeu de savoir mis à l'étude. II en ressort que la part empêchée de l'activité didactique, si elle permet de comprendre les raisons d'une éventuelle minoration des savoirs, oblige à problématiser la question de la construction de la référence en milieu difficile, en intégrant des échelles variables de temporalité au-delà même de la situation ou de la séance étudiée.

Descripteurs (TESE) : didactique, milieu défavorisé, éducation physique, enseignant, enseignement secondaire.

\section{LES DIFFICULTÉS DE L'ENSEIGNEMENT EN MILIEU DIFFICILE}

Si de nombreux rapports ont établi les inégalités scolaires persistantes et la faible efficacité des politiques éducatives territorialisées de compensation de ces inégalités, la question des pratiques d'enseigne- ment dans les milieux difficiles reste, comparativement aux analyses sociologiques et économiques, un domaine de recherche peu exploré (INRP \& Centre Alain-Savary, 2006). Quand il l'a été, la spécificité des pratiques didactiques et pédagogiques dans ces milieux a été plus particulièrement abordée sous l'angle de la pertinence des réponses apportées, eu 
égard aux enjeux d'apprentissage des élèves, notamment en mathématiques et en français (Amigues \& Kherroubi, 2003 ; Bautier, 2002 ; Butlen, PeltierBarbier \& Pezart, 2002 ; Kherroubi \& Rochex, 2004 ; Peltier-Barbier, 2004). Nous nous sommes intéressées dans le cadre de cette recherche à décrire et expliquer les formes spécifiques de l'activité didactique en milieu dit " difficile ». Nos travaux s'inscrivent délibérément dans une approche didactique des phénomènes d'enseignement car nous considérons que la diffusion des savoirs est au cœur de l'activité enseignante - y compris et plus encore - dans ces milieux.

Alors que se popularise insidieusement l'idée que la transmission des savoirs à l'école ne peut plus être au cœur des politiques éducatives dans certaines zones du territoire, alors que l'injonction à la " socialisation première " devient l'alpha et l'oméga de la plupart des propos bien pensants sur l'enseignement en milieu difficile (Johsua, 1999 ; Rochex, 1997), nous pensons que la part didactique de l'intervention enseignante reste méconnue et qu'il convient d'en examiner les conditions de possibilité pour rendre compte des formes inégalitaires de scolarisation en France (Terrail, 1997). Nous avons choisi de ne pas réduire la notion d'enseignement en milieu difficile à sa territorialisation, notamment en termes de zones d'éducation prioritaire (ZEP), réseau d'éducation prioritaire (REP), ou plus récemment de « réseau ambition réussite » (RAR), tant la difficulté du travail enseignant s'exprime en différents lieux, sous de multiples formes et registres.

\section{Quelques résultats récurrents relatifs aux recherches menées en milieu difficile}

De la lecture des travaux issus de différents champs de recherche sur l'enseignement en milieu difficile, nous retenons quelques constats marquants:

- les recherches de l'équipe ESCOL soulignent la difficulté perçue par les enseignants travaillant en ZEP qui se traduit par la mise en concurrence des visées de socialisation et d'apprentissage au détriment de ces dernières (Bautier, 2002; Bautier \& Rochex, 1997 ; Charlot, Bautier \& Rochex, 1992 ; Davisse \& Rochex, 1998 ; Kherroubi \& Rochex, 2002, 2004) ;

- les travaux de van Zanten (2001) sur l'école de la périphérie ont montré qu'en ZEP, l'écart entre ce qui est enseigné et les prescriptions des programmes est plus important que dans les établissements de centre-ville. Ils mettent en évidence un surajus- tement didactique aux caractéristiques des élèves autour de tâches simplifiées et de compétences de faible niveau ;

- récemment, des membres de l'équipe DIDIREM, se situant dans une approche didactique d'orientation ergonomique, ont mis en évidence en REP des gestes professoraux en mathématiques se traduisant par un éclatement des contenus enseignés sous forme de micro-tâches très individualisées et une absence de phase d'institutionnalisation des savoirs (Butlen, Peltier-Barbier \& Pezart, 2002; Peltier-Barbier, 2004). Ces constats seraient pour ces chercheurs à l'origine de malentendus didactiques semblables à ceux pointés par Bautier en français (2002) ;

- en EPS, quelques études révèlent des phénomènes similaires de réduction des contenus enseignés dans les établissements dit «difficiles » (Debars \& Amade-Escot, 2006 ; Poggi-Combaz, 2002).

Cette convergence des analyses, menées sous des cadrages théoriques variés, nous permet de conclure à la réalité d'un particularisme de l'enseignement en milieu difficile dont on peut considérer, avec différents auteurs, qu'il est, malgré les déclarations de bonnes intentions, politiquement autorisé voire même construit. Un des éléments remarquables que nous retenons de cette brève revue de la littérature est que l'enseignement en milieu difficile se traduit par une minoration des savoirs fondamentaux au bénéfice de savoirs plus superficiels et de savoir-faire sociaux. Cet état de fait n'est pas sans lien avec les difficultés rencontrées et ressenties par les enseignants exerçant dans ces contextes (Kherroubi \& Rochex, 2004 ; Saujat, 2001 ; van Zanten, 2001). Prendre en charge, au sein d'une problématique didactique, la réalité de la difficulté du travail du professeur nous a conduits à nous intéresser aux travaux qui, dans le domaine des sciences humaines et sociales, théorisent l'activité de I'homme au travail. La clinique de l'activité, en ce qu'elle « fait entrer dans l'activité de travail les conflits du réel qui opposent le sujet à lui-même [...] autour du concept d'activité empêchée [...] afin d'incorporer le possible et l'impossible dans l'activité » (Clot, 2001, p. 14), représente selon nous une perspective heuristique pour l'étude des pratiques didactiques des professeurs en milieu difficile.

\section{Les enjeux d'une étude \\ " du » didactique ordinaire en milieu difficile}

Lorsque Brousseau, à la fin des années 1960, posait les bases de la recherche en didactique en définissant l'enseignement comme « un projet social : celui 
de faire approprier à un élève un savoir constitué ou en voie de constitution » (Brousseau, 1998 (1), p. 51), la visée d'enseignement apprentissage (toujours d'actualité) était globalement soutenue par la société. Le projet politique défendu par/pour l'École et ses enseignants à cette époque portait la volonté affichée de l'accès de tous à une culture commune. L'objet de la théorie des situations didactiques, qui a influencé beaucoup de recherches (et soutenu de nombreuses innovations), était de modéliser par le biais d'une analyse systémique le jeu du professeur sur les jeux de l'élève confronté à un milieu didactique afin qu'aboutisse l'enjeu de savoir (pour un développement de la modélisation, voir Brousseau, 1998, p. 80-112). Les attaques institutionnelles portées depuis à l'école publique et à ses acteurs - attaques qui touchent aussi bien le niveau des textes des programmes que celui des lieux et structures, ou encore la définition du métier d'enseignant (Johsua, 1999) ne peuvent pas être sans effet sur la perception qu'ont les élèves et leurs professeurs de la tâche qui leur est prescrite. En ce sens, nous souhaitons pointer que l'évolution des conditions d'étude «du» didactique depuis le développement des ingénieries jusqu'à l'analyse plus contemporaine des situations en classe ordinaire n'est pas, selon nous, le résultat d'une simple évolution de la théorisation du domaine, mais résulte aussi de la nécessité de comprendre finement les processus inhérents aux changements des rapports aux savoirs au sein d'institutions didactiques particulières (comme en témoigne la synthèse de Schubauer-Leoni \& Leutenegger, 2005). Si le travail du professeur, qui vise comme le posait Brousseau à faire aboutir l'enjeu de savoir, rencontre aujourd'hui des empêchements de tout ordre, il est autre chose de dire, voire de banaliser l'idée, qu'il suffirait à l'enseignant de "tenir sa classe » pour qu'il ait réalisé sa tâche et ce même si l'institution, par ses injonctions implicites, pousse dans cette direction.

La spécificité de l'approche didactique, qui est d'étudier le fonctionnement du système du point de vue des savoirs en jeu, présente dans le contexte actuel des politiques éducatives une perspective d'analyse in situ (Amade-Escot, 2007) susceptible d'identifier les processus qui, dans les classes difficiles, permettront d'apporter les éclairages nécessaires à une École ne se satisfaisant pas de voir sa mission réduite à la seule socialisation normée.

Rejoignant le propos de Rochex (1997) selon lequel les ZEP, par l'effet de loupe qu'elles produisent, sont révélatrices d'enjeux relatifs à l'ensemble du système éducatif, nous pensons pouvoir nous faire une idée juste du fonctionnement de ce qu'est aujourd'hui l'École à travers l'observation du travail conjoint des professeurs et des élèves dans les milieux difficiles, en ce qu'il est potentiellement révélateur de ce que seront demain les conditions de ce même travail, quels qu'en soient les contextes. Un des enjeux premiers, nous semble-t-il, de l'étude du didactique ordinaire en milieu difficile est justement de comprendre et d'expliquer les processus à l'origine de difficultés génériques de l'enseignement et de l'apprentissage scolaires dans nos sociétés fragmentées. Cette mise au jour de possibles traits de généricité, quels que soient les contextes et/ou les disciplines, est au cœur de l'approche comparatiste en didactique. Dans ce cadre nous pensons contribuer, avec d'autres, à l'élaboration d'un modèle de l'action didactique qui, étant en mesure d'expliquer ce qui se passe en classe, ne risque pas « d'être une pure projection didacticienne " pour reprendre les mots de Schubauer-Leoni (2008). Nous suivons volontiers celle-ci dans la définition des enjeux du chantier dans lequel sont engagés les didacticiens : "Mettre au cœur de [la] problématique l'enjeu de savoir tout en tenant la question des élèves et donc [plus largement] des sujets articulée à cet enjeu de savoir, afin de continuer à montrer que le projet didactique mérite d'être poursuivi non seulement dans les classes mais aussi au sein des sciences humaines et sociales » (p. 86).

\section{CONSTRUCTION DE L'OBJET D'ÉTUDE : “L'ACTIVITÉ DIDACTIQUE EMPÊCHÉE »}

Pour prendre en charge la question des sujets afin de traiter cette articulation entre sujets et enjeu de savoir, nous avons choisi de recourir à la "clinique de l'activité » qui, au sein du champ de la psychologie, considère que l'activité réalisée n'est pas toute l'activité du sujet mais «l'actualisation d'une des activités réalisables dans la situation où elle voit le jour » (Clot, 2001). Le positionnement théorique de cet auteur permet de penser le réel de l'activité comme constitué également de "ce qui n'a pas pu se faire ", "ce qui ne s'est pas fait " et met en évidence la part “ contrariée ", « rentrée », "impossible» ou encore "empêchée » de l'activité de l'homme au travail. On peut dire que le concept d'activité empêchée permet de comprendre le développement de l'activité (évolution des buts, motifs et mobiles) et sa mise en souffrance. II nous semble que l'approche développée par la clinique de l'activité constitue un point de vue porteur d'éléments de compréhension des effets des milieux difficiles sur l'action didactique des ensei- 
gnants en classe. Nous défendons l'idée que la problématisation de l'activité professorale en termes "d'activité didactique empêchée » - qui consiste à décrire et à analyser ce qui s'enseigne/s'apprend en classe en s'attachant à la fois à la part réalisée et à la part empêchée de l'activité - donne accès à une compréhension générique de l'activité didactique des enseignants à même de renouveler l'analyse de l'enseignement et de ses difficultés, tout en permettant de rompre avec les discours d'impuissance du système ou d'accusation des enseignants.

Des recherches pionnières ont approché le travail de l'enseignant en mettant en évidence son " efficacité malgré tout » (Clot et al., 1998 ; Clot \& Soubiran, 1999 ; Roger, Roger \& Yvon, 2001). Mais dans I'ensemble, ces premières études minorent la dimension didactique, celle qui selon nous, reste le cœur de l'activité enseignante. Dans la période récente, d'autres travaux originaux ont ouvert des perspectives de rapprochement entre clinique de l'activité et didactique (Amigues, 2005 ; Lataillade, 2005 ; Saujat, 2001, 2004 ; Félix \& Saujat, 2008). Notre travail s'inscrit dans cette dynamique. II consiste, à partir d'un ancrage didactique, à s'intéresser aux conflits du réel de l'activité professorale pouvant être à l'origine de l'effacement des savoirs constaté dans l'enseignement usuel en milieu difficile.

\section{Convoquer "l'activité didactique empêchée » pour comprendre l'action didactique : protocole méthodologique}

L'enjeu épistémologique sous-jacent à cette étude a consisté à construire un "système de recherche ", c'est-à-dire un ensemble indissociable de protocoles et de concepts congruent avec la visée de la recherche (Leutenegger, 2003). Pour rendre compte du fonctionnement du système didactique en milieu difficile, nous avons mis en œuvre un dispositif d'observation du didactique ordinaire au sein duquel nous avons choisi d'introduire (pour accéder à l'activité didactique empêchée) un protocole inspiré de la clinique de l'activité. Le "système de recherche » retenu articule des données d'observation de séances et des données d'entretiens que nous explicitons et fondons dans les paragraphes suivants.

Les emprunts théoriques que nous avons choisi de faire à la " clinique de l'activité " dans la conception même de l'objet d'étude (l'activité didactique empêchée) ont imposé des aménagements afin de créer les conditions de production de matériaux pertinents et nécessaires au traitement des questions de recher- che. Concrètement, nous avions à résoudre les problèmes suivants : comment avoir accès à «l'activité didactique empêchée " des enseignants observés ? À partir de quel(s) matériau(x) ? Recueilli(s) de quelle(s) façon(s) ? Comment mener les analyses ? Ceci nous a conduits à élaborer une méthodologie originale.

Ce qui est au fondement de "l'activité empêchée ", selon les chercheurs en psychologie du travail, c'est qu'elle n'est pas objectivable à partir de la seule observation de ce qui est fait dans la tâche par le sujet. Dans la tradition de l'ergonomie de langue française, certains auteurs ont montré la nécessité d'une approche dialogique pour accéder au réel de l'activité (Clot \& Faïta, 2000). Divers protocoles d'entretien en autoconfrontation (simple et/ou croisée, méthode d'instruction au sosie) ont été développés, fondés sur la réalisation de l'activité par le sujet dans un autre contexte. Nous avons choisi de nous appuyer sur la méthode «d'instruction au sosie " (Clot, 2001; Oddonne, Re \& Briante, 1981) qu'il nous a fallu adapter et intégrer au protocole usuel d'observation du "didactique ordinaire" développé par Leutenegger (2003). Cette construction méthodologique, fondée sur des emprunts conceptuels assumés, a entraîné des modifications des dispositifs initiaux, tant celui de l'instruction au sosie que celui d'observation du didactique ordinaire (pour un développement, voir Monnier \& Amade-Escot, 2007).

\section{Modifications apportées}

\section{à la méthode d'instruction au sosie}

Rappelons rapidement la méthode d'instruction au sosie telle qu'elle est mise en œuvre en clinique de l'activité avant de pointer les principales modifications que nous y apportons. À l'occasion d'un entretien filmé, le sujet doit raconter à son sosie (le chercheur) un aspect de son travail à partir d'une consigne d'instruction rédigée par l'équipe de recherche; il doit veiller à communiquer au sosie tous les détails nécessaires lui permettant de le remplacer dans son travail. Un deuxième temps prévoit de faire commenter par le sujet par écrit la retranscription du dialogue avec le sosie.

Dans notre protocole de recherche, une première étape consiste à faire rédiger par l'enseignant observé l'instruction qu'il adresse à son sosie à partir d'une consigne d'instruction longue et orientée sur les aspects didactiques de la séance. Le sosie destinataire de l'instruction oriente l'accès au réel de l'activité par la résistance qu'il oppose au sujet instructeur (Clot, 1999). Dans notre étude, afin de permettre le développement d'un discours technique entre experts, nous avons opté pour un sosie qui soit un autre ensei- 
gnant d'EPS et non le chercheur didacticien. Ce choix tente de limiter les effets d'adressage du discours de l'enseignant à un autre type d'expertise (2). L'instruction est donc adressée par l'enseignant non pas au chercheur mais à un pair qui est en mesure de le remplacer lors de la séance suivante. Néanmoins, pour que le sosie puisse rendre à l'enseignant "sa propre activité étrangère » (Clot, 1999, p. 142-143) et mettre ainsi au jour, au travers du dialogue, la part empêchée de l'activité de ce dernier, nous avons sollicité, pour tenir le rôle de sosie, des enseignants dont le contexte de travail n'est pas le milieu difficile mais des établissements de centre-ville ou de banlieue «bourgeoise". Le sosie, parce qu'il est issu d'un contexte de travail différent, peut résister « en interposant entre l'instructeur et son action une image de la situation peu conforme à "l'idée" naturalisée que s'en fait le sujet " (Clot, 1999, p. 156). Le dialogue entre l'enseignant et le sosie, deuxième étape de notre protocole "d'instruction au sosie ", a lieu une fois que ce dernier a lu la consigne d'instruction écrite qui lui est remise et qu'il a pu préparer d'éventuelles questions.

L'entretien avec le sosie se déroule en présence du chercheur. Le rôle du chercheur consiste si besoin est, sporadiquement, à intervenir pour prolonger l'échange, à revenir sur des points évoqués ou trop rapidement abordés. Cependant, la conduite de l'entretien est à la charge du sosie qui initie le questionnement. Enfin une dernière étape, que nous appelons entretien post-protocole entre l'enseignant et le chercheur, se déroule après une première analyse par le chercheur des différents corpus de données. L'entretien post-protocole, deuxième contexte de réalisation, confronte l'enseignant aux traces du dialogue qu'il a eu avec le sosie, puis l'engage dans un échange avec le chercheur à propos des pistes d'analyse et d'interprétation élaborées par ce dernier.

Modifications apportées au dispositif d'observation du didactique ordinaire

Pour observer le didactique ordinaire, Leutenegger (2003) s'attache à construire ce qu'elle appelle une "clinique des systèmes ". Pour éviter le risque de naturalisation des observations, le dispositif formalisé par l'auteure articule un entretien ante-séance, un enregistrement vidéo de la séance, et un entretien post-séance. Les données d'observation constituent le corpus principal, les entretiens ante-séance et post-séance jouent le rôle de discutant des phénomènes observés. L'intégration de la méthode "d'instruction au sosie " au sein du protocole d'observation modifie en retour ce dispositif. Pour résumer, dans notre protocole, l'entretien ante-séance devient une discussion entre le chercheur et l'enseignant afin de recueillir des informations sur le cycle et de décider de la séance observée. Celle-ci, choisie en fonction du contenu d'enseignement, est filmée par le chercheur. L'entretien avec le sosie vient prendre la place de l'entretien post-séance, auquel s'ajoute l'entretien post-protocole (tels que décrits précédemment).

Pour conclure, ce protocole de recueil des données nous semble de nature à garder vive la problématique d'une clinique du système didactique (Leutenegger, 2003) au sens où il permet de questionner ce qui est considéré comme "signe " par le chercheur afin de saisir et de rendre compte de la dynamique évolutive qui préside au jeu du professeur sur le jeu des élèves, relativement au savoir mis à l'étude. Néanmoins l'introduction de l'instruction au sosie dans ce dispositif, en lieu et place de l'entretien post-séance, vient bousculer le statut des données d'observation en ce qu'il introduit la possibilité d'y associer une clinique de l'activité (au sens de Clot, 1999). Ainsi, ce n'est plus le corpus de la transcription de la vidéo qui initie l'analyse, mais un jeu d'allers-retours entre les corpus d'observation, d'entretien au sosie et d'entretien post-protocole.

Les données de ces entretiens permettent à la fois de questionner à nouveau la vidéo et de l'éclairer, sans que soit établi de chronologie entre l'utilisation des différentes pièces du corpus. Nous soutenons l'idée que l'introduction, dans le protocole d'observation du didactique ordinaire, d'un outil permettant la mise en enquête de l'activité crée des conditions méthodologiques utiles à la compréhension du fonctionnement du système didactique dans ses empêchements. Ainsi sans réduire la clinique - qu'elle soit des systèmes didactiques (Leutenegger, 2003) ou de l'activité (Clot, 1999) - à des aspects de méthode, nous considérons que l'articulation des perspectives impose des remaniements qui, du point de vue de chacun des cadres théoriques, peuvent être questionnables mais qui, vis-à-vis de notre problématique de recherche, ont pour intérêt de rendre possible l'investigation des phénomènes didactiques « ni (que) dans les savoirs ni (que) dans les sujets " comme nous y invitent Schubauer-Leoni et Leutenegger (2005, p. 408).

\section{Déroulement de la recherche}

Notre étude s'est déroulée sur quatre sites: deux collèges ZEP ou assimilés et deux lycées professionnels. Huit enseignants chevronnés d'EPS, quatre d'établissements difficiles (observés) et quatre 
d'établissements de centre-ville (les sosies) ont collaboré à notre recherche. Nous réalisons tout d'abord l'enregistrement de la séance en classe puis nous procédons aux différentes étapes de «l'instruction au sosie ». L'entretien post-protocole entre l'enseignant observé et le chercheur clôture le recueil des données. L'ensemble du corpus est composé de quatre entretiens ante-séance, quatre enregistrements vidéo, quatre entretiens sosie, quatre entretiens postprotocole et également de l'analyse a priori (3) de chacune des tâches proposées aux élèves dans les séances observées, des traces écrites des rédactions des instructions, des traces écrites des préparations des séances filmées et parfois de celles des séances précédentes.

Pour Leutenegger (2003), la prise en charge de ce qui fait «signe » pour le chercheur est rendue possible en assignant différentes fonctions aux traces recueillies. Au cours de notre recherche nous avons constaté que la confrontation du chercheur à ces traces, dès le moment de leur recueil, n'est pas anodine et influence les analyses qui en seront faites. En effet, le chercheur a vu la séance au moment de l'entretien avec le sosie. De la même manière il a participé à l'entretien avec le sosie lorsqu'il commence la transcription des données, et enfin, il a effectué une première analyse de l'ensemble du corpus avant de procéder à l'entretien post-protocole avec l'enseignant. Nous avons mesuré combien les impressions, voire les intuitions construites à partir d'indices, de fragments qui ont marqué notre esprit au fur et à mesure du déroulement de la recherche, peuvent orienter l'approche du corpus. Cette réalité du rapport du chercheur aux matériaux de la recherche nous a contraints (à la fois pour éviter toute surinterprétation et aussi pour enrichir l'analyse) à multiplier les allers et retours entre les entretiens et la vidéo au fur et à mesure de l'avancée dans l'analyse et l'interprétation. Le travail sur le corpus intègre ainsi les premières « impressions générales » au processus d'analyse pour, à chaque fois, orienter l'enquête vers les diverses pièces du corpus, mais aussi pour trouver les éléments qui viendront les conforter et/ou les infirmer au fil de l'analyse. Dégager la complexité de l'activité didactique des professeurs n'est jamais facile. En milieu difficile se surajoute de façon encore plus impérative une nécessaire "suspension du jugement". C'est dans ces allers-retours récurrents entre impressions générales et détails des traces recoupées que nous pensons pouvoir accéder et rendre compte de la part empêchée de l'activité didactique du professeur (4). Les résultats présentés dans la suite de l'article s'appuient sur quelques extraits d'un des quatre cas analysés. Ils mettent en évidence certains traits de l'activité empêchée d'une enseignante d'EPS lors d'une séance de badminton en lycée professionnel.

\section{ILLUSTRATION À PARTIR D'EXTRAITS D'UN CAS : L'ACTIVITÉ DIDACTIQUE EMPÊCHÉE DE NADIA (5)}

La séance observée est une séance de badminton conduite par Nadia avec une classe mixte de lycée professionnel. La tâche plus directement concernée par l'analyse présentée ci-après est la première de la séance. Elle relève, selon Nadia, de ce qui est communément appelé “l'échauffement ». Il s'agit d'une situation hybride dans laquelle l'enseignante, pour des raisons que l'analyse du cas mettra au jour, tente à la fois d'échauffer physiquement ses élèves tout en introduisant un enjeu de savoir nécessaire à la chronogenèse (6) de cette séance.

Les élèves sont quatre par terrain : un d'un côté, trois de l'autre. Ces trois joueurs doivent frapper le volant à tour de rôle après un déplacement imposé : ils doivent en effet rentrer sur le terrain par un espace d'un mètre, matérialisé par deux plots posés au sol, appelé la «porte ». Cette « porte » est située au milieu de la ligne de fond du court (à 6,70 $\mathrm{m}$ du filet). L'élève sur le terrain opposé a pour consigne de renvoyer le volant en direction des trois joueurs. Le but de la tâche est de parvenir à faire le maximum d'échanges. L'analyse a priori de la tâche prescrite aux élèves permet d'en décrire les enjeux. II s'agit pour les élèves de construire une posture de frappe du volant leur permettant à la fois de maintenir l'échange avec le joueur placé sur le terrain adverse et de s'inscrire dans un déplacement vers l'avant. Les enjeux de savoir dans cette tâche sont les suivants: adapter son déplacement en fonction de la trajectoire du volant initiée par le joueur adverse, anticiper le placement pour le renvoyer, s'équilibrer au moment de la frappe. II s'agit d'optimiser la rencontre du tamis de la raquette avec le volant, ce qui a déjà été travaillé lors des séances précédentes (comme l'indiquent les traces écrites de la préparation de séance). La visée de ce dispositif associe un travail de la technique (frappe du volant avec la raquette) à un enjeu d'échauffement. La variable de commande du dispositif au principe du double enjeu de la tâche (travail de la frappe et échauffement) réside dans la distance entre la «porte " et le lieu où sera frappé le volant. L'élément décisif de la réussite de la tâche est en relation avec le déplacement à effectuer avant et/ou après la frappe du volant. La «porte » est supposée baliser un trajet entre deux points. L'enseignante n'a déterminé qu'un point de passage obligé : la porte 


\section{Extrait 1 de l'entretien sosie}

\begin{tabular}{|c|c|l|}
\hline 19 & $\mathrm{~S}$ & $\begin{array}{l}\text { alors par rapport à cette une heure quinze tu me dis y a une pression temporelle importante } \uparrow \text { c'est } \\
\text { c'est pas suffisant pour cette classe une heure quinze motrice } \uparrow\end{array}$ \\
\hline 20 & $\mathrm{P}$ & $\begin{array}{l}\text { ben disons que je je // je la compare avec heu un travail qu'on peut faire en bac professionnel où on les } \\
\text { [a trois heures }\end{array}$ \\
\hline 21 & $\mathrm{~S}$ & $\begin{array}{l}\text { [hum } \\
22\end{array}$ \\
\hline $\mathrm{P}$ & $\begin{array}{l}\text { et où comme on va sur ces mêmes installations } \uparrow \text { ça fait une séance qui dure heu qui dure deux heures } \\
\text { et faire des rétroactions } \uparrow \text { en ayant le sentiment que je perds pas mon temps et que je continue de faire } \\
\text { avancer et que au plan moteur y a toujours un temps d'activité qui est assez important } \uparrow\end{array}$ \\
\hline 23 & $\mathrm{~S}$ & $\begin{array}{l}\text { d'accord } \\
\end{array}$
\end{tabular}

d'entrée sur le terrain, située sur la ligne de fond de court. Elle n'a pas positionné d'autres plots obligeant un déplacement long (par exemple un plot en zone avant sur le côté du terrain). Elle n'a pas non plus contraint le joueur adverse à jouer des volants courts ce qui aurait pu, d'une autre manière, forcer les élèves à produire un déplacement plus important avant et après la frappe. Le dispositif tel qu'il est construit et présenté aux élèves ne semble pas relever d'un milieu didactique agencé pour que les élèves s'engagent dans un déplacement permettant a priori de réaliser l'échauffement physique escompté. II semble qu'ils peuvent réaliser le but de la tâche sans mettre en cause leur équilibre et en n'effectuant que de courts déplacements. Que dit l'enseignante à propos de ce dispositif au sosie ? Que se passe-t-il lors de l'action conjointe professeurs élèves en classe?

\section{Les enjeux de savoir débordent l'enjeu identifié par l'analyse a priori}

Nous nous intéressons aux données relatives à ce court épisode pour mettre au jour en quoi la part empêchée de l'activité didactique est susceptible de rendre compte d'un certain effacement des savoirs mis à l'étude. Dans les extraits qui suivent, nous n'utilisons pas les marques de ponctuation de l'écrit mais nous indiquons tous les signes du codage liés à la prosodie (en gras : les variations accentuelles ; // : les brefs silences ; xxx : les segments inaudibles ; $\uparrow$ et $\downarrow$ : l'augmentation ou la diminution de hauteur de la voix ; [: les chevauchements). Les éléments sur lesquels nous appuyons l'analyse sont distingués en caractères italiques afin de faciliter la lecture de nos interprétations. À gauche de chaque extrait, apparaissent les tours de parole dans la transcription des entretiens. Nous commençons l'analyse par les indices prélevés dans l'entretien sosie. Le sosie (S) engage l'entretien avec Nadia $(P)$ en la questionnant sur une des informations communiquées dans l'instruction qu'elle a rédigée, information relative à la durée de la leçon.

Nadia exprime le sentiment de se trouver dans une contrainte temporelle forte avec cette classe. Le sosie paraît s'étonner de l'urgence exprimée par l'enseignante alors que la classe bénéficie quand même d'1 h 15 de temps de travail. Cette remarque pousse l'enseignante à préciser qu'elle se sent dans l'urgence parce qu'elle se trouve en situation de devoir gérer des objectifs qui lui paraissent concurrentiels: "proposer des situations d'apprentissage » et " faire des rétroactions ", et ce «tout en ne perdant pas trop de temps " pour garantir une quantité de pratique suffisante. Cette répartition du temps porte un enjeu particulier ancré dans la perception qu'a l'enseignante de ses élèves (tour de parole 29) : " c'est heu c'est un public qui a envie $\downarrow$ qui a envie de pratiquer $\uparrow »$. Le croisement de ces indices avec l'analyse de la vidéo de la séance montre que le temps de parole et de mise en place pour chaque exercice est relativement long (de 4 à 8 minutes). Sur l'ensemble de la séance le temps de pratique physique des élèves représente seulement la moitié du temps total de la séance. Nous considérons que cet indice révèle une certaine difficulté de l'enseignante à garantir à ses élèves un temps de pratique physique important alors même qu'elle exprime cette préoccupation dans l'entretien sosie.

Plus tard ( $3^{\mathrm{e}}$ et $4^{\mathrm{e}}$ minute), le sosie questionne Nadia sur le choix de son échauffement. Notons que l'enseignante ne fait pas d'échauffement traditionnel (usuellement quelques minutes de course dans le gymnase) mais commence par la tâche décrite plus haut. Compte tenu du début de leur échange, le sosie imagine que ce choix s'appuie sur la pression temporelle précédemment évoquée. Mais l'enseignante le coupe et répond qu'il y a en fait deux raisons à ce choix. 


\section{Extrait 2 de l'entretien sosie}

\begin{tabular}{|l|c|l|}
\hline 42 & S & donc c'est la pression temporelle encore $\downarrow$ qui guide un [peu ton \\
\hline 43 & $\mathrm{P}$ & [à la fois la pression temporelle $\uparrow$ et puis quand même le public que j'ai face à moi quoi $\downarrow$ \\
\hline 44 & $\mathrm{~S}$ & et le public qui n'adhèrerait pas à un échauffement \\
\hline 45 & $\mathrm{P}$ & $\begin{array}{l}\text { ah je suis pas sûre que sur les deux tours de gymnase que je proposerais ils ne feraient pas // heu la } \\
\text { moitié en marchant heulou qu'ils seraient pas en train de faire autre chose } \downarrow\end{array}$ \\
\hline
\end{tabular}

\section{Extrait 3 de l'entretien sosie}

\begin{tabular}{|l|l|l|}
\hline 51 & $\mathrm{P}$ & $\begin{array}{l}\text { ben disons/que heu je démarre par de la coopération parce que heu comme je fais pas justement } \\
\text { d'échauffement classique } \uparrow \text { il me semble que c'est un peu risqué si de suite je dis heu y a de l'opposi- } \\
\text { tion on cherche à marquer des points surtout qu'eux ils sont } \downarrow \text { davantage sur un jeu où ils utilisent la } \\
\text { force heu/force du bras etc. } \uparrow \text { plutôt que que la finesse } \uparrow \text { si je leur dis de suite qu'on est dans l'oppo- } \\
\text { sition/bon je je fais courir à l'élève/un certain risque physique donc heu }\end{array}$ \\
\hline
\end{tabular}

Plus loin, au tour de parole 51, l'enseignante précise que pour échauffer physiquement les élèves et éviter les risques de blessure, l'exercice qu'elle met en place démarre par une phase de coopération.

Cet extrait de l'entretien sosie souligne les conflits d'intention auxquels l'enseignante doit faire face et combien elle mobilise, pour le faire, le fruit de son expérience. Nadia se trouve en effet confrontée à une tâche délicate : échauffer physiquement ses élèves en évitant à la fois un bras de fer - qu'elle pense très probable si elle proposait un échauffement traditionnel sous forme de course - et les risques de blessures susceptibles de se produire si elle engageait les élèves dans un match d'échauffement. C'est la résolution de cette alternative qui l'amène à choisir ce dispositif susceptible, selon elle, de concourir à la fois à l'échauffement et à introduire un enjeu d'apprentissage en lien avec le reste de la séance. Elle propose néanmoins un jeu de coopération plutôt qu'un jeu de confrontation (un match) qui bien que pouvant plaire aux élèves risque, en raison de l'intensité de l'opposition, d'être source d'accidents. À ce stade de l'analyse, on perçoit déjà que cette enseignante a développé des usages professionnels dans un contexte contraint, ou tout du moins au sein duquel elle isole et repère des contraintes qui orientent ses choix didactiques. Se pose alors la question de savoir si l'enseignante parvient à échauffer efficacement ses élèves. Ici le retour à la bande vidéo devient essentiel pour donner chair aux indices d'empêchement suggérés par l'entretien sosie.

\section{Mise au jour de tensions contradictoires}

Dans ce que dit l'enseignante aux élèves pour présenter le travail demandé on remarque une sorte d'inconfort, mais aussi une certaine lucidité sur les conséquences du compromis qu'elle a "choisi » de faire. Voici les consignes de cette enseignante présentées dans un extrait de la bande vidéo :

$\mathrm{P}$ (s'adressant à toute la classe, $3^{\mathrm{e}}$ minute de la séance) : "On va décomposer en deux moments, premier moment vous êtes en situation d'échauffement donc au lieu de courir autour de la salle ça vous sert d'échauffement vous allez être en coopération, premier but : échanger le maximum de volants en situation de un contre trois d'accord, on va faire ça pendant 4 minutes, à l'issue des 4 minutes on considérera que vous êtes échauffés hein par rapport à l'utilisation de la raquette "

Nadia commence en annonçant clairement aux élèves que l'exercice proposé remplace les tours de terrains usuels (dont on a vu aux tours de parole 45 et 51 de l'entretien sosie qu'ils pourraient, selon elle, rebuter les élèves). Puis, énonçant qu'elle considérera que les élèves sont échauffés au bout de quatre minutes, ce qui à l'évidence est insuffisant, elle coupe sa phrase. Cette dernière (formellement adressée aux élèves) est ponctuée par le "hein » que nous interprétons à la lumière de l'ensemble du verbatim de la séance et des entretiens comme un indice révélateur chez cette enseignante d'un trouble, d'une pensée connexe ou encore d'un contrôle sur ce qu'elle est en train de dire et qui précise (peut-être à l'attention du 
chercheur observateur de la séance ?) que ce qui aura été réellement travaillé dans ce laps de temps est moins l'échauffement physique des élèves que la prise de repères dans la frappe du volant : " hein par rapport à l'utilisation de la raquette». Ces indices révèlent selon nous que Nadia n'ignore pas quelles sont les conditions à réunir pour échauffer les élèves. Nous considérons que le croisement des données de l'entretien sosie avec celles de l'observation didactique "fait signe » (au sens de Leutenegger, 2003) et permet d'interpréter cet épisode en termes de conflit d'intention, conflit alimenté par le souci de mobiliser les élèves (ne pas les rebuter). Cette tâche dite "d'échauffement " est également porteuse d'un second enjeu : reprendre un savoir déjà abordé dans les séances précédentes sur la frappe du volant, parce qu'il est nécessaire à la situation d'apprentissage suivante qui portera sur « frapper le volant pour varier les trajectoires » (minutes 45 à 67 de la séance). Mais les enjeux de savoirs prévus dans la suite de la séance viennent en partie en perturber l'économie, comme le pointe l'analyse qui suit.

En effet, cette analyse de la vidéo montre qu'il faudra plus de deux minutes après l'explication de l'exercice pour mettre en place le cadre du travail (le milieu initial), minutes aux cours desquelles l'enseignante veille au respect de l'aménagement matériel demandé (la " porte "), "vous avez pris les plots pour faire la situation ou pas ? ", et à la répartition des joueurs sur les terrains, « non on est 3 d'un côté et 1 de l'autre, 3 et 1 ! Tu es là Pierre, heu Julien [...] Où est le troisième avec vous ? Où est le troisième ? " Pendant ce temps les élèves jouent librement en faisant fi des consignes.

Nous pointons également que le temps effectif de pratique des élèves est faible. L'exercice dure, comme prévu, seulement 4 minutes. En termes d'échauffement, il convient cependant d'en retirer tous les déplacements marchés effectués par les élèves pour ramasser le volant et se replacer sur la zone d'engagement (la zone du terrain où on effectue le premier coup d'envoi qui s'étend de la ligne de fond jusqu'à une ligne située à $2 \mathrm{~m}$ du filet). Les meilleurs élèves (au nombre de 4) parviennent à faire jusqu'à 50 frappes consécutives, ce qui représente une certaine continuité dans l'action et montre que ces élèves ont (re) trouvé leurs sensations de frappe du volant. En revanche, pour l'ensemble de la classe, les déplacements courus sont pratiquement inexistants contrairement aux attentes de l'enseignante. En nous attardant sur ses interventions au cours de la réalisation de l'exercice, on repère qu'à la $11^{\mathrm{e}}$ minute elle rappelle la consigne à certains élèves peu dynamiques (extrait vidéo) : " mais tu rentres obligatoirement par la porte [...] Tu dois être en mobilité ". Un peu plus tard en s'appuyant sur les productions d'élèves qui jouent assez long et dans l'axe, avec des déplacements très réduits (extrait vidéo), Nadia s'adresse à la classe entière et, parlant à la cantonade, réoriente ses consignes sur la variation des trajectoires : "Si ça marche bien on essaie d'allonger les trajectoires! " Cet indice confirme notre interprétation précédente, il s'agit bien d'une avancée chronogénétique qui a pour visée de préparer les élèves à la situation qui va suivre (minute 45) et qui assigne aux élèves le but de gagner l'échange dans un jeu d'opposition contre un partenaire, en envoyant le volant dans des zones particulières du terrain, ce qui suppose de produire des trajectoires variées en longueur et en direction.

Enfin, à la $4^{\mathrm{e}}$ minute, en réaction aux comportements des élèves qui jouent des volants hauts et courts (extrait vidéo), Nadia précise encore, pour toute la classe, la nature de ce qu'il y a à faire : "C'est un échauffement sur les trajectoires les plus précises possibles!" Ces éléments d'observation nous amènent à considérer que l'enseignante abandonne progressivement l'objectif d'échauffement physique, d'autant que les deux consignes successives (des trajectoires longues, puis précises) ont potentiellement pour effet de réduire encore la distance à couvrir par les élèves qui, rappelons-le, doivent entrer par le fond du terrain ("la porte») pour jouer la balle. L'action de l'enseignante tend à privilégier les ajustements posturaux favorables à la production de trajectoires variées en introduisant une modification du milieu, engageant de fait une avancée chronogénétique par rapport à l'enjeu de la situation suivante.

Se confirme ici ce qui avait été évoqué dans l'analyse a priori de la tâche: l'agencement du milieu didactique ne permet pas, dans ce tout début de séance, d'engager les élèves dans le déplacement escompté (seul moyen de les échauffer physiquement, comme l'auraient fait des tours de terrain). Toutefois, il nous paraît risqué d'interpréter cela en manque de savoir didactique de l'enseignante. Nous considérons plutôt que la réalité du dispositif mis en œuvre et la gestion qui en est faite rendent compte de la résolution de tensions (au sens de "l'efficacité malgré tout» selon Clot, 1999) auxquelles est confrontée l'enseignante, tensions amplifiées (selon les moments) par le public avec lequel elle doit composer et par les objectifs qu'elle fixe à sa séance.

Des interrogations demeurent pourtant. Pourquoi cette enseignante, qui craint d'imposer à ses élèves 
des tours de terrain, prend-elle le risque de leur faire faire une situation d'échauffement à la fois contraignante et éloignée d'un simple jeu (susceptible d'obtenir leur adhésion) et dont la mise en place s'avère coûteuse en temps et en énergie ? Y aurait-il un enjeu plus fort que le simple échauffement physique pour lequel l'enseignante est prête à prendre le risque de se confronter au refus des élèves ? Revenons sur les données issues de l'entretien sosie. L'extrait 4 , à la $2^{\mathrm{e}}$ minute de l'entretien, permet nous semble-t-il d'affiner l'interprétation.

L'enseignante, dans cet extrait, revient sur une temporalité antérieure à la séance qui tisse l'activité didactique au-delà même de l'action observée. La première séance du cycle qu'évoque Nadia a consisté en une succession de matchs visant le classement des élèves selon leurs résultats. Cette première séance, classique dans l'enseignement scolaire du badminton, est assez peu contraignante en matière d'apprentissage. II s'agit d'une entrée dans le cycle à forte dimension ludique. L'incise de l'enseignante, au tour de parole 33 de l'extrait 4, souligne selon nous son souci de ne pas laisser penser aux élèves qu'ils pourraient jouer librement à chaque séance du cycle comme lors de la première séance. Ces «pré-occupations » (au sens de Clot, 1999), qui s'imposent à l'enseignante et confèrent du sens à son travail, pèsent dans la construction du dispositif mis en place pour débuter la séance. Plus loin dans l'entretien avec le sosie, Nadia confirme notre analyse lorsqu'elle pointe le décalage entre ce qu'elle propose aux élèves dans les séances et leurs représentations de la discipline EPS : « donc pour l'instant $\uparrow$ comme je suis déjà en dehors de leur représentation à travers les situations que je propose $\uparrow$ j'ai l'impression que heu j'alourdirais [...] » (tour de parole 99 de l'entretien sosie). Se précise ici combien ce professeur assume ses choix d'intervention, tout en estimant qu'ils demandent un effort à ses élèves pour adhérer au travail proposé. Nous pensons que le sentiment construit au fil de la pratique quotidienne vient faire obstacle à d'autres possibles: “j'ai l'impression que heu j'alourdirais ». Pour autant, Nadia ne renonce pas, en témoignent, à la minute 46 , vers la fin de l'entretien avec le sosie, sa position et sa volonté affirmées de ne pas renoncer à ses convictions et à sa conception de l'EPS et de l'apprentissage.

On mesure ici la façon dont Nadia, prenant l'ascendant lors de l'échange avec le sosie, marque son point de vue en énonçant avec force son refus du renoncement, justement à propos de ce qui est estimé absolument nécessaire à l'apprentissage et qu'elle évoquait déjà dès le début de l'entretien sosie : " faire des situations d'apprentissage, des rétroactions et garantir une quantité de pratique suffisante " (tour de parole 22, extrait 1 de l'entretien sosie). Tout un uni-

\section{Extrait 4 de l'entretien sosie}

\begin{tabular}{|l|l|l|}
\hline 33 & $\mathrm{P}$ & $\begin{array}{l}{[\ldots] \text { je renie pas le fait que comme je veux les faire évoluer } \uparrow \text { et que je veux pas xxxxx parce que c'est }} \\
\text { vrai que pour eux/je pense queeee } \uparrow \text { jouer comme ils jouent en première séance } \uparrow[\ldots] \text { [des matchs à } \\
\text { visée diagnostique ont constitué l'essentiel de la première séance du cycle] }\end{array}$ \\
\hline 34 & $\mathrm{~S}$ & hum hum hum \\
\hline 35 & $\mathrm{P}$ & $\begin{array}{l}{[\ldots] \text { du coup ça les heu/heu ça les amène à se dire on pourrait jouer comme ça sans contrainte/en jeu }} \\
\text { liiibre heu sans que vous veniez nous embêter avec des situations particulières } \uparrow\end{array}$ \\
\hline
\end{tabular}

\section{Extrait 5 de l'entretien sosie}

\begin{tabular}{|l|l|l|}
\hline 313 & $\mathrm{P}$ & $\begin{array}{l}\text { alors c'est vrai } \uparrow \text { que moi d'entrée sur la situation d'échauffement c'est déjà une situation qu'est une } \\
\text { situation d'apprentissage puisqueee }\end{array}$ \\
\hline & & {$[\ldots]$} \\
\hline 332 & $\mathrm{P}$ & $\begin{array}{l}\text { // bien quand même 'fin parce que moi jeee/je pense qu'on peut attendre quand même des acquisitions } \\
\text { avec quand [même }\end{array}$ \\
\hline 333 & $\mathrm{~S}$ & [oui oui [mais \\
\hline 334 & $\mathrm{P}$ & [avec un apprentissage structurééé heu avec heu j'ai pas envie de renoncer à ce type de chose $\uparrow$ \\
\hline 335 & $\mathrm{~S}$ & d'accord d'accord \\
\hline
\end{tabular}




\section{Extrait 6 de l'entretien sosie}

\begin{tabular}{|c|l|l|}
\hline 49 & $\mathrm{P}$ & $\begin{array}{l}\text { oui oui } \downarrow \text { je pense que et par rapport } \downarrow \text { au badminton par rapport à cette APS-là } \uparrow \text { et pour l'avoir } \\
\text { vérifié avec d'autres élèves } \downarrow \text { heu heu avec d'autres classes } \downarrow \text { d'autres années } \downarrow / \text { il me semble qu'il } \\
\text { vaut mieux démarrer comme ça } \downarrow \text { [par la coopération] je crois [sourire] }\end{array}$ \\
\hline & & {$[\ldots]$} \\
\hline 55 & $\mathrm{P}$ & je reste dans cette [situation]... c'est vrai que... et je l'ai ritualisée $\uparrow$ \\
\hline
\end{tabular}

vers de délibération et de négociation entre ce qui donne sens aux possibles du métier (transmettre des savoirs malgré tout) et les possibles de l'action en contexte et qui oriente selon nous les choix de l'enseignante tant au niveau micro du dispositif, qu'au niveau plus large de la conception de l'ensemble de la séance et du cycle.

Au cours de l'entretien post-protocole nous sommes revenues avec l'enseignante sur son début de séance. Nous lui avons demandé de lire plusieurs extraits de l'entretien sosie relatif au thème de l'échauffement, extraits au cours desquels nous avions pointé le fait qu'elle semblait perturbée par ce que lui proposait le sosie (« engager les élèves dans une logique de compétition ") et auquel elle n'avait pas semblé réagir à ce moment là. Suite à une controverse (minutes 3 à 6) sur la place du duel ou des défis entre élèves dans l'échauffement, et relativement aux propositions du sosie visant à engager les élèves dans une logique de compétition, Nadia se positionne par les paroles suivantes (voir extrait 6 de l'entretien sosie).

Au tour de parole 49, l'enseignante ponctue sa phrase avec une intonation descendante à chaque locution, ce qui peut indiquer qu'elle est pensive ou retenue. Cette impression de doute ou de réserve est amplifiée par le «je crois » et le sourire qui concluent sa réponse au sosie. La proposition du sosie, relative à la gestion de l'opposition et de la coopération dans l'échauffement et qui consiste à proposer aux élèves d'établir des records par deux à comparer soit à leur propre record de la séance précédente, soit à ceux de leur camarade (tours de parole $48 \mathrm{~S}$ et $50 \mathrm{~S}$ ), trouble visiblement l'enseignante qui répond au sosie par une phrase chaotique, où l'on peut repérer que la pensée défile en même temps que le discours s'interrompt : " je reste dans cette... c'est vrai que... je l'ai ritualisée $\uparrow »$. Au cours de l'entretien post-protocole, après avoir été invitée par le chercheur (C) à lire les extraits de la transcription de l'entretien avec le sosie relatif à ces passages (tours de parole 42 à 55 de l'entretien avec le sosie), Nadia $(P)$ revient sur cet échange.
Ce qui est dit dans l'entretien post-protocole nous éclaire encore. Le dispositif construit et proposé par l'enseignante aux élèves pour l'échauffement mais aussi pour commencer la séance s'ancre tout d'abord dans la perception que s'est forgée Nadia de ses élèves, peu dociles et peu enclins à accepter les contraintes "d'un apprentissage structuré " (tour de parole 334, extrait 5 de l'entretien sosie). Forte de cette perception, ce moment de la séance défini par l'enseignante comme un «moment-clé » doit lui permettre à la fois d'échauffer les élèves (même si on a pu voir que finalement cet enjeu était minoré) mais surtout de poser des conditions au principe de son activité didactique : celui de faire entrer les élèves dans un cadre de travail qui, quelles que soient les séances, les implique dans des situations structurées au sein desquelles ils auront à prendre à leur charge la construction du savoir visé. II s'agit pour l'enseignante de construire des règles de fonctionnement qui vont au-delà même de l'enjeu de savoir que le dispositif peut porter et que nous avons décrit dans l'analyse a priori. Nous pensons trouver dans ces indices des éléments nous permettant de considérer que le professeur, en inscrivant le processus d'enseignement apprentissage dans une temporalité qui dépasse celle de l'action didactique immédiate, ouvre des possibles parfois sous-estimés par les analyses locales que nous discutons maintenant.

\section{L'activité didactique entre empêchement et ingéniosité}

L'enquête menée à partir du cas de Nadia permet de décrire son activité dans ses empêchements et son ingéniosité. II apparaît de façon remarquable que cette enseignante gère dans une unité de temps court (celle de la tâche) divers enjeux :

- ne pas entrer en conflit avec les élèves dès l'entame du cours ;

- asseoir l'EPS comme une discipline d'enseignement dans laquelle il faudra travailler et non pas simplement s'amuser ;

- parvenir à faire travailler les élèves quitte à s'opposer à leurs représentations de l'EPS ; 


\section{Extrait de l'entretien post-protocole}

\begin{tabular}{|c|c|c|}
\hline 41 & C & $\begin{array}{l}\text { bon je résume donc tu me dis tout à l'heure de mémoire que ça a un peu interpellé ta conception de } \\
\text { l'échauffement donc c'était justement une question que je voulais te poser puisque justement à la } \\
5^{\mathrm{e}} \text { minute de l'entretien tu émets un doute sur le choix que tu as fait de démarrer par un échauffement } \\
\text { raquette et volant }\end{array}$ \\
\hline & & {$[\ldots]$} \\
\hline 43 & C & donc j'aimerais que tu lises cette partie-là \\
\hline 44 & $\mathrm{P}$ & $\begin{array}{l}\text { je suis effectivement en train de réfléchir à ce début d'échauffement parce que je pense qu'il n'y a } \\
\text { aucune certitude que c'est toujours la phase de doute tout comme on ne sait pas // on n'a pas de } \\
\text { certitude pour savoir comment on rentre dans l'activité il y a cette part de doute y a aussi la part de } \\
\text { doute qui existe à comment débuter la séance } \uparrow \text { comment heu // parce que c'est c'est un moment-clé/ } \\
\text { de la séance } \uparrow\end{array}$ \\
\hline \multirow[t]{2}{*}{45} & C & clé par rapport à quoi par rapport au climat par rapport au contenu par rapport à quoi ? [...] \\
\hline & & {$[\ldots]$} \\
\hline 50 & $\mathrm{P}$ & $\begin{array}{l}\text { Je ne sais pas si j'arriverais si je laissais les élèves beaucoup plus libres au tout début de la séance } \\
\text { sans placer autant de consignes d'entrée, etc. } \uparrow \text { je ne sais pas dans quelle mesure moi en tant qu'en- } \\
\text { seignante je serais en mesure de les rattraper 'fin rattraper de les replacer ensuite dans mon mode de } \\
\text { fonctionnement voilà } \uparrow\end{array}$ \\
\hline
\end{tabular}

- ne pas renoncer à ses convictions concernant son rôle et sa mission d'enseignant;

- parvenir à garder le contrôle de la classe ;

- échauffer physiquement ses élèves.

La diversité et la multiplicité des enjeux qui se superposent révèlent le "réel de l'activité » de l'enseignante. On peut dire que Nadia n'échappe pas aux constats posés par les cliniciens de l'activité : elle agit "dans une interférence des intentions " (Clot, 1999 , p. 19). Nous ajouterons que l'activité didactique empêchée, mise au jour à l'occasion de cet extrait, opère dès la construction de la tâche dans le temps de travail privé de l'enseignante dont l'analyse a priori nous a permis de pointer les limites. Mais l'examen que nous en avons fait, dans l'imbrication des multiples intentions à l'œuvre, nous amène à conclure que «l'empêchement» s'enracine aussi dans l'expérience acquise et la naturalisation des conditions d'exercice du métier. Nous ne concluons donc pas au manque de savoir didactique de l'enseignante comme pourrait le laisser penser une étude isolée de la tâche. La difficulté, voire l'impossibilité, de circonscrire l'activité didactique des professeurs à l'échelle de temps et d'analyse qui serait celle de chaque dispositif invite à en considérer la complexité : d'une part, en envisageant l'aboutissement de l'enjeu de savoir comme devant être instruit à la lumière des différentes échelles temporelles dans lequel il se trouve tissé (Leutenegger, 2008 ; Schubauer-Leoni et al., 2007) ; d'autre part, en ne sous-estimant pas les empêchements susceptibles de se produire à une échelle temporelle plus réduite. Nous considérons en effet que ces derniers sont des marqueurs de ce qui - dans l'action didactique réalisée - renvoie au réel de l'activité (au sens de Clot, 1999). En paraphrasant cet auteur, nous pensons avoir produit une " mise en enquête de l'activité (ses motifs et ses mobiles) à partir de l'action" (ici didactique), nous ajoutons que l'action didactique se trouve en retour éclairée par la mise au jour de l'activité empêchée. Ainsi, dans la séance de Nadia, le premier dispositif - qui permet que la séance soit possible (les élèves acceptent de rentrer dans le travail proposé) - se traduit localement par l'effacement de sa fonction d'échauffement physique sans pour autant que l'enjeu de savoir relatif à l'affinement de la frappe du volant puisse être véritablement mis à l'étude. Mais ce dispositif concourt aussi, grâce à l'usage qui est installé, à ce que l'enseignante puisse mener avec ses élèves des discussions denses en didactique avec une écoute attentive des élèves comme le mettent en évidence d'autres moments de cette même séance, non présentés dans le cadre de cet article. Ce constat souligne que la construction d'une référence conjointe en tant qu'émergence d'expériences jugées compatibles avec des pratiques sociales (Schubauer-Leoni, 2008), ici relatives au badminton, nécessite, si l'on veut en saisir la dynamique, de ré-articuler différentes temporalités. II indique aussi que l'action didactique en ce 
qu'elle actualise le travail enseignant dans toute l'épaisseur de son activité (y compris ses empêchements) est essentiellement faite de récupération (7).

\section{DISCUSSION : L'ACTION DU PROFESSEUR POURSUIT UN HORIZON LOINTAIN QUI DÉPASSE L'ACTION ELLE-MÊME}

La recherche dont nous venons de rendre compte - à travers les trop brefs extraits d'un des cas étudiés souligne ce que Brousseau avançait à la fin des années 1970 à propos de «l'observation des activités didactiques " : "L'utilisation d'une observation exige toujours un effort pour discerner ce qui était possible dans ce qui ne s'est pas produit et ce qui est nécessaire dans ce qui s'est produit " (Brousseau, 1978, p. 133). L'auteur indique que " cet effort est une construction scientifique ". Cette citation nous permet de discuter en quoi l'approche didactique peut s'enrichir - sous réserve de conversions (voir Monnier \& Amade-Escot, 2007) - des problématiques de la clinique de l'activité qui, d'une autre manière et avec d'autres fondements, soutient que l'activité déborde la tâche. Dans l'étude de cas présentée, l'activité didactique déborde la tâche d'enseignement en ce qu'elle est au carrefour d'une construction collective (dont on prend la mesure à l'occasion des débats de métier lors du dialogue avec le sosie) et d'une signification donnée à ce même métier par le professeur en référence à des exigences de savoirs (le souci de ne jamais renoncer à ce que les élèves s'engagent dans des apprentissages spécifiques du badminton pardelà les difficultés rencontrées). Les importations que nous avons faites depuis la didactique à la clinique de l'activité nous ont permis d'enrichir l'analyse didactique du travail enseignant tout en instaurant un dialogue entre deux orientations de recherche (8). Grâce aux conversions opérées lors de l'insertion de la problématique de l'activité empêchée dans la théorisation didactique, nous avons pu envisager quelques éléments de réponse à la question de l'efface- ment des savoirs en milieu difficile. Une des réponses apportées est d'envisager le jeu du professeur sur le jeu des élèves en termes d'action conjointe et selon un point de vue délibérément ternaire, prenant en considération l'analyse épistémique des enjeux de savoirs (Sensevy \& Mercier, 2007). Ce point de vue souligne combien la construction de la référence dans ces contextes comme ailleurs relève de temporalités multiples et emboîtées susceptibles de cristalliser des tensions et des conflits de "pré-occupations" (Clot, 1999) à l'origine de l'empêchement de l'activité professorale. Ainsi les quatre études de cas que nous avons menées montrent que les pratiques enseignantes peuvent être abordées en maintenant à la fois le fil très fort de l'attachement à ce que peut et doit être l'École, y compris en milieu difficile (participer à la transmission d'une culture), et le fil de la complexité des enjeux ou des motifs d'agir du professeur au travail. II pourrait sembler que nous rejoignons alors Saujat (2001) lorsqu'il conclut que l'action du professeur ne peut pas être comprise « jusqu'au bout si l'on postule qu'ils [les choix] sont uniquement [nous soulignons] finalisés par une recherche de pertinence didactique » (p. 95). Nous suggérons que la recherche dont tente de rendre compte cet article, si elle confirme la complexité des motifs d'agir professoraux, montre au contraire que l'action didactique, éclairée en retour par la convocation des concepts de la clinique de l'activité, n'est intelligible que si l'on prend en compte l'attachement du professeur à "l'objet d'horizon » lointain, dépassant l'action didactique elle-même tout en lui donnant sens : celui qui est justement de rendre possible l'aboutissement de l'enjeu de savoir (9) et qui, eu égard aux empêchements qui y sont associés, est source de difficultés, voire parfois de souffrance au travail.

Nathalie Monnier nmonnier2@wanadoo.fr

DIDIST, CREFI-T, université Paul-Sabatier-Toulouse 3 , Université de Toulouse

Chantal Amade-Escot

DIDIST, CREFI-T, université Paul-Sabatier-Toulouse 3, Université de Toulouse 
(1) La référence de 1998 renvoie à l'ouvrage qui rassemble les différentes publications de Guy Brousseau. La question de la didactique en lien avec le projet social de l'école apparaît très tôt dans la pensée broussaldienne et est clairement exprimée dès sa thèse d'état en 1986.

(2) Nous avons bien conscience que cette façon de procéder transforme sensiblement le cadre méthodologique initial de l'instruction au sosie. Cette modification est selon nous nécessaire au traitement des questions de recherche dans la perspective et la posture épistémologiques que nous adoptons. La substitution du chercheur par un autre enseignant se justifie par ailleurs dans le but d'atténuer à cette étape du recueil de données ce que Chevallard identifie comme «les demandes non officielles mais pressantes des acteurs permanents du système " à l'adresse du didacticien, dont il considère qu'elles sont consubstantielles de la situation de recherche didactique (Blanchard-Laville, Chevallard \& Schubauer-Leoni, 1996, p. 44). Il s'agit donc d'une conversion méthodologique que nous assumons.

(3) En didactique l'analyse a priori est un outil méthodologique permettant « de mettre au jour l'intentionnalité portée par le dispositif, en quoi il peut devenir un milieu pour l'étude et enfin d'identifier quels sont les savoirs en jeu " (Amade-Escot, 2007, p. 24). Cette analyse permet également d'en identifier les variables de commande, de guider la lecture faite de la gestion par l'enseignant du savoir en jeu et d'anticiper les réponses possibles des élèves. L'analyse a priori est essentielle en ce qu'elle rend possible la confrontation des réponses des élèves à l'analyse épistémique ainsi réalisée. Précisons toutefois « qu'elle [est] conçue comme [une] méthode qui permet de construire l'analyse, de poser les questions, d'interroger, à la condition d'éviter le rabattement de l'activité sur le modèle théorique qu'[elle] fournit " (Sensevy \& Mercier, 2007, p. 204).

(4) Nous ne sous-estimons pas le fait que la recherche menée ait pu produire un développement professionnel pour les enseignants qui ont bien voulu y participer. Nous en avons, par ailleurs, rendu compte avec l'une d'entre eux lors d'un symposium traitant des relations entre chercheurs et praticiens (Falga \& Monnier, 2008). Dans le cadre de notre recherche, cet aspect qui n'a pas été au centre de la problématisation de notre objet d'étude marque une posture épistémologique différente, eu égard à celle adoptée en clinique de l'activité.

(5) II s'agit d'un pseudonyme.

(6) La chronogenèse définit ce qui a trait à l'avancée des savoirs au fil de la temporalité didactique. Elle dépend pour l'essentiel de l'action enseignante mais peut également être portée par certains élèves. Dans la théorie de l'action conjointe en didactique, la chronogenèse est nécessairement articulée à ce qui a trait à l'évolution des responsabilités respectives du professeur et des élèves (topogenèse) et à l'évolution d'un milieu initial proposé par l'enseignant potentiellement porteur du travail des élèves (mésogenèse). Ces trois descripteurs (chronogenèse, topogenèse gésogenè trois dé introduits par Chevallard (1992). (1992). Ils sont actuellement développés par les didacticiens comparatistes (Sensevy, Mercier \& Schubauer-Leoni, 2000 ; Sensevy \& Mercier, 2007).

(7) Nous pensons ici retrouver ce qui est au cœur de l'activité enseignante en tant que travail humain caractérisé par un " état de dysfonctionnements à la fois permanent et invisible de l'extérieur [...] [et qui] porte à considérer que récupérer une situation qui dérive est maintenant au cœur des activités professionnelles » (Clot, 1995, p. 191).

(8) Dialogue conceptuel dont rendent compte plusieurs publications (Monnier \& Amade-Escot, 2007 ; Félix \& Saujat, 2008).

(9) Cette conclusion pourrait être considérée comme elliptique. Nous la soutenons parce que la question du maintien des enjeux de savoirs en milieu difficile est politiquement vive quoique parfois sous-estimée, comme l'ont montré les travaux que nous citons en introduction de cet article ; l'étude des quatre cas de notre recherche confirme qu'il s'agit bien, pour les enseignants, de l'horizon qui donne sens à leur travail. Nous considérons néanmoins avec d'autres didacticiens et aussi d'autres ergonomes que les relations entre action didactique conjointe et activité doivent continuer à être instruites. Cela suppose une discussion épistémologique que nous menons par ailleurs, mais que le cadre nécessairement restreint de cet article ne permet pas de conduire à la hauteur de l'exigence qu'elle requiert en termes de développement argumentaire.

\section{BIBLIOGRAPHIE}

AMADE-ESCOT C. (2007). Le didactique. Paris : Éd. revue EPS.

AMIGUES R. (2005). "Les dispositifs d'aide aux élèves en difficulté comme révélateurs de l'activité enseignante ". In L. Talbot, Pratiques d'enseignement et difficultés d'apprentissage. Toulouse : Éd. Érès, p. 105-116.

AMIGUES R. \& KHERROUBI M. (2003). "Les pratiques de la classe en "milieux difficiles"”. Recherche et formation, $\mathrm{n}^{\circ} 44$.

BAUTIER É. (2002). « L'enseignement en ZEP et les recherches en didactique du français ". Revue française de pédagogie, $\mathrm{n}^{\circ} 140$, p. 53-64.

BAUTIER É. \& ROCHEX J.-Y. (1997). "Apprendre: des malentendus qui font la différence ». In J.-P. Terrail, La scolarisation de la France. Critique de l'état des lieux. Paris : La Dispute, p. 105-122.

BLANCHARD-LAVILLE C., CHEVALLARD Y. \& SCHUBAUERLEONI M.-L. (1996). Regards croisés sur le didactique.
Un colloque épistolaire. Grenoble : Éd. La Pensée sauvage.

BROUSSEAU G. (1978). "L'observation des activités didactiques ". Revue française de pédagogie, $\mathrm{n}^{\circ} 45, \mathrm{p} .130-$ 139.

BROUSSEAU G. (1998). Théorie des situations didactiques. Grenoble : Éd. La Pensée sauvage.

BUTLEN D., PELTIER-BARBIER M.-L. \& PEZART M. (2002). "Nommés en REP, comment font-ils ? Pratiques de professeurs d'écoles enseignant les mathématiques en REP ». Revue française de pédagogie, n 140, p. 41-52.

CHEVALLARD Y. (1992). "Concepts fondamentaux de la didactique: perspectives apportées par une approche anthropologique ". Recherches en didactique des mathématiques, vol. 12, $\mathrm{n}^{\circ} 1$, p. 73-112.

CHARLOT B., BAUTIER É. \& ROCHEX J.-Y. (1992). École et savoir dans les banlieues... et ailleurs. Paris : Armand Colin. 
CLOT Y. (1995). Le travail sans l'homme ? Pour une psychologie des milieux de travail et de vie. Paris : La Découverte.

CLOT Y. (1999). La fonction psychologique du travail. Paris : PUF.

CLOT Y. (2001). "Clinique de l'activité et pouvoir d'agir ». Éducation permanente, $\mathrm{n}^{\circ}$ 146, p. 7-16.

CLOT Y. \& FAÏTA D. (2000). «Genre et style en analyse du travail. Concepts et méthodes ". Travailler, $\mathrm{n}^{\circ} 4$, p. 7-42.

CLOT Y. \& SOUBIRAN M. (1999). "Prendre la classe : une question de style ? " Société française, $n^{\circ} 62$, p. 78-83.

CLOT Y., MICHAUX B., ROGER J.-L. \& SANGLA S. (1998). «Enseigner malgré tout. Table ronde sur le métier enseignant ». Société française, $n^{\circ} 61$, p. 32-39.

DAVISSE A. \& ROCHEX J.-Y. (1998). Pourvu qu'ils apprennent... : face à la diversité des élèves. Le Perreux-surMarne: CRDP de l'académie de Créteil.

DEBARS C. \& AMADE-ESCOT C. (2006). « Enseigner le badminton à une classe de primo-arrivants : l'incidence du contexte ZEP (zone d'éducation prioritaire) sur la pratique d'intervention ". eJRIEPS, $\mathrm{n}^{\circ}$ 9, p. 35-43. Disponible sur Internet à l'adresse : <http://www.fcomte.iufm. fr/CD_RENTREE_2007/rentree/RECHERCHE/ejrieps/ ejournal9/Debars\%20(f)eJ9.pdf> (consulté le 4 décembre 2009).

FALGA N. \& MONNIER N. (2008). « L'activité didactique en milieu difficile: intérêt d'un collectif de recherche élargi ». Communication orale au symposium "Situations d'interactions entre enseignants et chercheurs en éducation physique : quelles collaborations? ", $5^{e}$ biennale de I'ARIS, Rodez.

FÉLIX C. \& SAUJAT F. (2008). «Faire classe en dehors de la classe: une nouvelle professionnalité enseignante ?» Les dossiers des sciences de l'éducation, $n^{\circ} 20$, p. 123-136.

INRP \& CENTRE ALAIN SAVARY (2006). Apprendre et enseigner en " milieux difficiles ». Lyon : INRP.

JOHSUA S. (1999). L'école entre crise et refondation. Paris : La Dispute.

KHERROUBI M. \& ROCHEX J.-Y. (2002). « La recherche en éducation et les ZEP en France. $1^{\text {re }}$ partie: Politique ZEP, objets, postures et orientations de recherche ». Revue française de pédagogie, $\mathrm{n}^{\circ} 140, \mathrm{p} .103-131$.

KHERROUBI M. \& ROCHEX J.-Y. (2004). « La recherche en éducation et les ZEP en France. $2^{\mathrm{e}}$ partie : Apprentissages et exercice professionnel en ZEP : résultats, analyses, interprétations ". Revue française de pédagogie, $\mathrm{n}^{\circ} 146$, p. 115-181.

LATAILLADE G. (2005). L'activité des professeurs de mathématiques dans les dispositifs d'aide en classe de $6^{e}$. Analyse didactique et analyse du travail : quelles conditions pour un dialogue? Thèse de doctorat non publiée, sciences de l'éducation, université de Provence-AixMarseille 1.

LEUTENEGGER F. (2000). "Construction d'une "clinique" pour le didactique. Une étude des phénomènes temporels de l'enseignement ". Recherches en didactique des mathématiques, vol. 20, $\mathrm{n}^{\circ} 2$, p. 209-250.

LEUTENEGGER F. (2003). «Étude des interactions didactiques en classe de mathématiques: un prototype méthodologique ". Bulletin de psychologie, vol. 56, $\mathrm{n}^{\circ} 4$, p. $559-571$
LEUTENEGGER F. (2008). "L'entrée dans une code écrit à l'école enfantine et l'articulation entre le collectif et l'individuel: comparaison de deux études de cas ". Éducation \& didactique, vol. 2, $\mathrm{n}^{\circ} 2$, p. 7-42.

MONNIER N. \& AMADE-ESCOT C. (2007). « L'activité didactique empêchée: construction méthodologique entre science didactique et science du travail». Actes $d u$ congrès de I'AREF, Strasbourg. Disponible sur Internet au format PDF à l'adresse : <http://www.congresintaref. org/actes_pdf/AREF2007_Nathalie_MONNIER_370. pdf $>$ (consulté le 4 décembre 2009).

ODDONNE Y., RE A. \& BRIANTE G. (1981). Redécouvrir l'expérience ouvrière. Vers une autre psychologie du travail. Paris : Éditions sociales.

PELTIER-BARBIER M.-L. (2004). Dur d'enseigner en ZEP. Dur pour les élèves, dur pour les enseignants. Grenoble : Éd. La Pensée sauvage.

POGGI-COMBAZ M.-P. (2002). «Distribution des contenus d'enseignements en EPS au collège selon les caractéristiques sociales du public scolaire: des différences non aléatoires ». Revue française de pédagogie, $\mathrm{n}^{\circ} 139$, p. 53-69.

ROCHEX J.-Y. (1997). « Les ZEP, un bilan décevant ». In J.-P. Terrail, La scolarisation de la France. Critique de l'état des lieux. Paris : La Dispute, p. 123-140.

ROGER R., ROGER J.-L. \& YVON F. (2001). " Interrogations pour une analyse de l'activité enseignante ». Éducation permanente, $\mathrm{n}^{\circ} 146, \mathrm{p} .115-125$.

SAUJAT F. (2001). «Co-analyse de l'activité enseignante et développement de l'expérience: du travail de chacun au travail de tous et retour ». Éducation permanente, $\mathrm{n}^{\circ} 146$, p. 87-98.

SAUJAT F. (2004). «Transformer l'expérience pour la comprendre: I'analyse du travail enseignant ». In J.-F. Marcel \& P. Rayou (dir.), Recherches contextualisées en éducation. Paris : INRP, p. 79-89.

SCHUBAUER-LEONI M.-L. (2008). « La construction de la référence dans l'action conjointe professeur-élève ". In N. Wallian, M.-P. Poggi \& M. Musard (dir.), Co-construire des savoirs : les métiers de l'intervention dans les APSA. Besançon: Presses universitaires de Franche-Comté, p. 67-86.

SCHUBAUER-LEONI M.-L. \& LEUTENEGGER F. (2005). «Une relecture des phénomènes transpositifs à la lumière de la didactique comparée ". Revue suisse des sciences de l'éducation, vol. 27, n 3, p. 407-429.

SCHUBAUER-LEONI M.-L., LEUTENEGGER F., LIGOZAT F. \& FLUCKIGER A. (2007). «Un modèle de l'action conjointe professeur-élèves : les phénomènes didactiques qu'il peut/doit traiter ». In G. Sensevy \& A. Mercier, Agir ensemble. L'action didactique conjointe du professeur et des élèves. Rennes: PUR, p. 51-91.

SENSEVY G. \& MERCIER A. (2007). Agir ensemble. L'action didactique conjointe du professeur et des élèves. Rennes : PUR.

SENSEVY G., MERCIER A. \& SCHUBAUER-LEONI M.-L. (2000). "Vers un modèle de l'action didactique du professeur. À propos de la cours à 20 ». Recherches en didactique des mathématiques, vol. $20, \mathrm{n}^{\circ} 3$, p. 263-304.

TERRAIL J.-P. (1997). La scolarisation de la France. Critique de l'état des lieux. Paris: La Dispute.

VAN ZANTEN A. (2001). L'école de la périphérie. Scolarité et ségrégation en banlieue. Paris : PUF. 